\section{Richard Webber}

was until July of last year managing director of the UK Micromarketing operations of Experian. The originator of both ACORN and MOSAIC, one of his current interests is the application of segmentation to interactive contact channels.

\section{New Technology Briefing}

\section{Customer recognition systems: A prerequisite for effective CRM?}

\author{
Richard Webber
}

Received (in revised form): 22 January 2002

\begin{abstract}
Today it is common for most large enterprises, when contemplating a major enhancement of their CRM capability, to consider their requirements primarily in terms of software functionality, systems and data integration and change management. Raising standards of data quality, including for example the accuracy and currency of customer names and addresses, has typically been considered either unrelated to CRM or of secondary importance to the data integration element of CRM.

This may now be beginning to change. In the USA, many organisations with the largest volumes of outbound direct mail and inbound call centre traffic are now beginning to adopt external systems for what are variously described as 'identity linking', 'customer recognition' and customer data integration ('CDI'). In addition to performing routine address matching, these systems match, standardise, verify and update name and address records against a centralised universal register of known names and addresses.

The significance of such systems, it would appear from early implementations, goes far beyond improving data quality. Proponents — vendors and users - increasingly see these linking technologies as having a crucial impact on the design of the data management systems on which their CRM systems rely.

This paper investigates the origins and significance of these new systems, explains how they differ from traditional 'merge-purge' and address management services, provides examples of how they may be changing traditional data management processes and considers how likely it is that their use will spread to countries other than the USA.
\end{abstract}

\section{Context - The importance of address recognition in CRM} The annual level of spend on CRM technology is enormous - in the USA alone, according to industry commentators, ${ }^{1}$ an amount totalling some 0.5 per cent of the entire economy will be spend on outsourced CRM services by 2004. In the UK it has been calculated that call centres now employ more people than the car, coal and textile industries combined.

Despite this investment there is much anecdotal evidence to suggest that many consumers have a very negative view of their experience with 


\section{Recognition and customer satisfaction}

\section{Building a $360^{\circ}$ view}

\section{The entity recognised}

those contact points in large organisations whose operation is driven primarily by CRM systems.

Clearly not all of this dissatisfaction can be blamed on the technology itself. Consumers get fed up with levels of staffing which result in excessive waiting time on the telephone and with the poor training in IVR (interactive voice recognition) design which results in inappropriate messages. Nevertheless a significant amount of dissatisfaction results from inaccuracies in the raw data which drive the CRM systems.

In a single-product business selling through a single channel, small errors in name and address data quality are often immaterial. The post still gets through. But in a complex organisation, where the customer's name and address is used to link together information from disparate data sources, quite small inaccuracies in name and address details can lead to a quite serious loss of accuracy in the overall customer view.

The core argument at the heart of most CRM systems is that a customer relationship can be more efficiently managed - for the benefit of customer and supplier alike - when the contents of separate, previously disparate operational databases are consolidated together to provide a single ' $360^{\circ}$ view' of that customer. The richness of this view will be greatest where information from operational systems is enhanced with recent contact history as well as proprietary or 'pooled' information available from external sources.

Problems with the use of the name and address to consolidate these information sources accurately occur in four areas: discrepancies in the manner in which the name and address is originally captured; variations in the entity for which the match is required; lack of notification of changes of address; and the handling of complex situations where, for example, one customer may use more than one address or where one address may relate to more than one consumer.

Basic differences in the recording of an address originate from many sources. Where names and addresses are taken down over the phone it is easy for 'phonetic' spelling errors to occur, particularly with the name and postcode elements. When addresses are taken down locally, suburb names are often added for convenience and town names abbreviated. For aspirational reasons many consumers prefer not to use the form of their address as designated by the postal authorities, and most suppliers consider it judicious to address customers in their preferred style rather than in that approved by the postal authorities.

Depending on the nature of the business, the entity that needs to be recognised for matching purposes may be the address itself, as for example with an energy supplier whose product is supplied to a particular location. Or the entity that needs to be recognised may be a person, as for example in the instance of a credit card operator. There are occasions where the requirement for recognition may operate at both address and person levels, as for example with a bank which may have multiple relationships with different individuals at the same address, some of whom may be joint account holders.

With 17 per cent of US residents and 10 per cent of UK residents 


\section{Recognising complex identities}

\section{Establishing a customer match key}

\section{Matching internal and external files}

moving house each year, prompt updating of current and previous address details is also a requirement of a fully watertight customer recognition system. This is a particular problem for organisations, such as life assurance companies, with a long average interval between customers' transactions.

In addition to the issue of the entity being matched - address, person or both - there are situations where more than one address may be required for accurate recognition. As in Spain and Sweden, where over 10 per cent of consumers now have a second home, a growing number of customers may record more than one home address with their suppliers. With the growth of private landlords in the UK, there are businesses such as utility providers which may want to be able to match on the basis of who owns as well as who occupies a property.

The seriousness of these issues tends to be masked where an organisation is matching together disparate internal files, and where these files contain information pertaining to transactions with existing customers, since these typically rely on a customer number having already been generated by the organisation to provide an effective match key. Even where a multi-product organisation relies on a central file to access the relationship between this customer number and the account numbers of the various products and services that any particular customer has used, this customer number will originally have been set up using the name and address supplied by the customer and will still be linked to it. That name and address will be used both as a match key to external data sources and to recognise customers when they use channels, such as the phone, where no visible or electronic identification of their customer number is available.

Where addresses are matched to externally sourced data, for instance in list rental applications, 'scored' using external overlay data and screened against suppression files (for example do not mail, fraud, gone away and mortality files), the internal customer number is no use. Accurate address recognition is also critical for identifying whether an enquirer is an existing customer, whether at a current or previous address, a lapsed customer or someone who has never been a customer, and what if any recruitment mailings that person may recently have received.

Until recently the two options an organisation would be faced with were whether to develop their own in-house systems for address recognition and matching or whether to purchase and tailor for their own use an externally developed address recognition software product.

The external option, it increasingly appears, will in future split between a software solution and some form of online $\mathrm{ASP}^{2}$ If an online service is adopted then a reference-file-linked CDI system provides an attractive alternative to basic address matching. Access to this register improves the accuracy and efficiency of the initial address recognition process, provides an encrypted numeric match key to enable lower-cost matching of files in-house and enables the organisation to use the ASP operator's services to identify and track customers whose address details may have changed. 


\section{The emergence of consumer information providers}

\section{Data management challenges}

\section{How CDI/linkage originated as a commercial service}

CDI is a business proposition which has been promoted in the USA since 1999. By far the largest provider of CDI services is the database management company Acxiom. Acxiom, which operates out of Little Rock, Arkansas, has over 100 licensed users of its CDI ASP, most of them among the higher-volume mailers in the USA. ${ }^{3}$

During 2001 CDI services have also been launched by the two leading credit reference/information services organisations in the USA, Experian and Equifax, the latter working in concert with Fair Isaacs, the leading US builder of credit scoring systems.

Over the years Acxiom, Experian and Equifax have each specialised in the creation and commercial exploitation of gigantic databases of information on private individuals. The data contributing to these databases have been sourced from public and private sources. These include information from vehicle registrations, telephone subscriber books, housing valuations, lifestyle surveys, mail-order lists and rented mailing lists, post office files, credit account information, public information on debtors etc.

According to their source and the constraints imposed by legislation, these databases have been used by clients to evaluate the creditworthiness of existing and new customers, to obtain qualified prospects for new business development, to append overlay data to existing customer files and to screen prospect mailings.

Given the volume of address-related information that they handle and the amount of address matching that the provision of these services entails, it is not surprising that all three organisations have made very significant investments in address recognition and matching technology, primarily as suppliers of bureau operators rather than as software vendors.

Particularly among organisations most reliant on these sources of information, such as mail-order firms and banks, it became apparent it would be more cost-effective to outsource the operational management of many of these repetitive data management functions to data suppliers in order to get access to the most current information available on their proprietary databases.

The requirement to minimise costs of repeated processing of these files led each supplier independently to implement a number of innovations, many of which were initially introduced as back-room processing procedures rather than as externally marketable services. For example, to avoid the cost of matching each client file to a whole series of reference files, the names and addresses on the reference files are consolidated into a single master file. Each name and address is then given a unique numeric key (whence the term ID linkage), which is then appended to records on the contributing files.

By screening client files against the register of addresses that contains this unique numeric key, it becomes possible to do a number of things. Where data protection permits the merging of the different files, screening against separate reference files can be undertaken as one single process rather than as a series of sequential operations. Where it does not, the search against the separate files is greatly speeded up because of the 
Unofficial match keys emerge

\section{Initial ROI justifications for CDI}

use of a consistent, accurate and continuously updated numeric match key.

The construction of a single master file is also used to maintain information on name and addresses for validation purposes, on gone aways and on changes of address. The single master file also provides greater security, in that it enables the service provider to set flags indicating access rights for each user to different information sources.

The construction of a single master file also enabled the bureau processors to assign their own 'unofficial' unique numeric key both to individual consumers and to private households. While initially these identifiers were used solely for internal purposes, it gradually became apparent that there were significant benefit to CRM users for these identifiers to be made available to them in encrypted form.

A system designed originally for internal purposes, subsequently revealed to and shared with a small number of major clients, was identified by Acxiom as a potential (and very real) source of competitive marketing advantage, hence the packaging up and launch in 1999 of the 'Abilitech' brand. Abilitech provides access to these reference identifiers in encrypted format both as part of a wider range of outsourcing database management services and as a generic ASP product. According to Acxiom this product requires a lower level of customisation to integrate with a CRM solution than name and address software products provided by companies specialising solely in packaged software.

'Productising' their capabilities has made the existing data management capabilities of Acxiom, Experian and Equifax accessible to a wider audience within the direct marketing and CRM industries and set the framework for enabling enhanced data quality within a much wider range of organisations.

\section{New paradigms for processing and updating CRM base files}

Investment in CDI systems is often justified initially in terms of lower processing costs and/or quality improvements. As with other aspects of CRM it was originally difficult to develop formal rules for predicting return on investment (ROI) in data quality enhancement. However, by offering to undertake preliminary data quality audits, vendors have been able to benchmark the quality of potential users' data against the average of competitors in their sector. Based on these audits, Acxiom has estimated that 90 per cent of high-volume mailers can achieve a return on investment of 12:1 from CDI data quality improvements, even when taking into account solely direct mail applications. ${ }^{4}$

In the USA, the requirement to comply with new legislation can also be a catalyst for investment in CDI. One such instance cited by Experian ${ }^{5}$ is the requirement to comply with a federal statute, know as the GrammLeach-Bliley statute, which makes it mandatory for a financial services organisation to inform its customers about their marketing activities and to give them the right to avoid or opt out of these marketing contacts.

Opportunities for cost reduction occur in three areas. Where the repeated matching of large files is required, savings may be available 


\section{Recognition at the customer set-up stage}

\section{Applying segmentation to real time channels}

through the use of numeric rather than address match keys. In addition savings on postage are achieved because it has consistently proved more accurate when deduplicating two files to match each of them separately to a common master reference file than to search for similar-looking names on the two files separately. A third cost saving occurs in situations where name and address changes can be notified as part of the customer recognition process.

Experian cite, for example, a global telecoms operator for whom the application of ID linkage to an entire database delivered a 10 per cent reduction in undeliverable mail and a 20 per cent reduction in data management costs due to the elimination of duplicate records.

To some organisations (BMC software and Mercedes are quoted by Acxiom in this respect), reduced annoyance to valued customers from the mailing of inappropriate information is considered a more important 'saving' than the reduction in wasted mail.

Whereas it is fairly straightforward to achieve cost reductions without significant process modification, and to achieve these in a first-phase project, the quality improvements inherent in improved matching typically require more long-term redesign, usually involving the introduction of new data transfer processes.

One key improvement is in new customer recognition. Once an existing customer database has been fully ID linked, and the customer IDs added to the master reference file, the new customer registration process can be modified to undertake a search of the integrated customer file/master reference file using the linkage codes. This can be used not just to identify with greater accuracy whether a prospect is an existing customer at his/ her current address, but to identify whether that prospect is a previous customer at the same address, an existing or previous customer whose details are held at an old address or a prospect who has, or has not, been mailed. Both these processes can be used to enable the determination of the current customer number, where it exists, or the allocation of a new customer number where it does not. Such systems are typically designed to ensure that the registers of customer identifiers held by the ASP provider and by the client are updated consistently and in real time.

The availability of a consistent easy-to-match 16-digit code also makes possible in practice what many CRM systems claim to make possible but often only deliver in theory, namely the rapid and affordable delivery of highly segmented customer contact strategies to a variety of different points of customer interaction. The benefit of the match key is not just that it facilitates the retrieval of these contact codes, but that it makes it very much quicker and simpler to update the strategies in real time to take into account unpredicted customer events as and when these occur. According to Carroll, ${ }^{6}$ these are the benefits which in the long term are likely to contribute the highest returns on investment, although their implementation is likely to lag behind the achievement of reduced direct mail costs and improved customer satisfaction through greater accuracy of customer data.

Where contributors pool information, there is interest in the use of the CDI system for verification, giving a score for each new prospect on 


\section{Improved cost efficiency in data management}

\section{Recognising changes of address}

dimensions such as level of activity (ie how many other members of the pool does the prospect trade with) and recent of activity (over how long a period have transactions been undertaken with the individual). Such information has been demonstrated in the UK as being particularly effective in combating Internet fraud. ${ }^{7}$

Where customer files and universal registers share common name and address identifiers (allowing for encryption), there are clear productivity and speed improvements in matching customer data against a proprietary and pooled database whose confidential nature means that they cannot be passed on to the client. Examples of such files are credit reference datafiles, registers of fraudsters, mailing preference scheme members, mortality registers, gone aways and registers of name and address changers.

Whereas originally the same names and addresses on the client file were repeatedly matched against the same names and addresses on the proprietary registers, the use of CDI systems enables the matching process to be undertaken at marginal costs of up to 50 per cent less. With intelligent thought it also becomes possible and certainly attractive to use the system to process only occurrences containing some form of change flag.

For example, it becomes possible to restrict screening to existing customers against new/changed entries on the proprietary registers and new/changed customers against all entries. Given the low volatility of customer and proprietary file records, such matching may amount to less than 10 per cent of the original matching task based on a monthly update cycle.

Another very attractive use of CDI systems is for the ASP provider to supply a proactive notification service to the client whereby the client is informed on a real-time basis of all the numeric identifiers on his customer database for which recent additional or altered information may have been obtained by the service provider. For example, an insurance provider could be informed for which of his customers a notification of change of address had been received, a mail-order client the numeric identifiers of those customers and previously mailed prospects for whom a 'gone away' flag had been received, and a bank a change in demographic data covered by a lifestyle survey. Such services are particularly attractive as the focus of the CRM system moves away from campaign-based mailing programmes with their fixed-interval timetable to event-based communication strategies based on 'trigger' information.

Clearly the development of applications of this sort has to be undertaken within the data protection and fair processing frameworks of domestic governments and, where appropriate, with the consent of clients and their customers.

Inevitably, both in the USA and the UK, there are many instances where existing guidelines provide no clear rulings as what is or is not permissible. Likewise, many potential as well as existing users of CDI systems are working through in their own minds what they consider to be acceptable or unacceptable uses of their own data.

However, with the potential to organise data flows between 
The diffusion of CDI

\section{Competition between vendors}

organisations and CDI suppliers in new ways, CDI systems need ideally to be designed into the overall CRM project rather than adopted as part of a second-phase 'data quality' initiative.

\section{What is the future for generic numeric customer identifiers in the USA?}

The current view in the USA is that the preoccupation with the implementation of CRM systems has distracted attention from issues such as data quality. Notwithstanding efforts to recruit IT vendors as strategic partners, the majority of Acxiom's first 100 Abilitech licences have arisen primarily through direct discussions with their clients, rather than through conferences, seminars and the recommendations of database consultants and other intermediaries. It is therefore somewhat of a hidden revolution and one which, for obvious reasons, vendors have been happier to pilot with a limited number of established key clients on a customised project basis. Nevertheless the design of the Acxiom product is engineered deliberately to support remote, generic application and to be bolted into modular CRM designs. Users are welcome to but not necessarily required to use Acxiom as a data management outsourcer.

As client experience in CRM matures, there is recognition among all vendors that data quality issues are moving closer towards the centre of the stage and that issues of cost containment, particularly through not mailing deceased, gone aways and movers, is seen as a sure return on investment. But the vendors are also coming to terms with the fact that many managers responsible for supporting CRM applications are principally IT specialists and, compared with the direct marketing specialists who would be responsible for traditional direct-mail databases, are relatively ignorant of data quality issues which are taken for granted by traditional direct marketing organisations. Ignorance rather than lack of demonstrable return on investment is felt to be the chief limit to adoption of the business application.

For the moment it makes sense for vendors to concentrate sales effort on a small number of large organisations, particularly those which have regular and frequent online contact with their customers. All are happy to leave the development of the medium-tier market to such time as experienced consultants are available to incorporate their own expertise in this area as part of the package of services they provide to individual clients. It is not so much that delivery needs to be routinised as that each implemention still requires substantial consultancy input.

Barriers to entry are seen to be significant. With three major organisations involved in the promotion of systems, alliances with developers of in-house software, such as Harte Hanks, Trillion or QAS, is seen to be more likely than competition from them. The fact that competing vendors to Acxiom are entering the market is, in many respects, beneficial to Acxiom, since there is an obvious fear on the part of major users that they could become 'locked in' to the system of a single supplier. 


\section{Factors following US diffusion}

\section{Will CDI systems appear in Europe?}

Despite the plethora of commercially significant innovations in direct and database marketing that have originated in the USA, it is still difficult to distinguish which new innovations 'will travel' and which will not. Of those that do travel, many find the Canadian, the UK and other Englishspeaking markets easier to penetrate than continental Europe. Maybe these are the markets that American firms use to test whether their concepts will travel.

Compared with other markets, the construction of effective CDI systems based on reference databases is made easier in the USA both by the comparative ease of access to large public domain datasets and by the absence of a single unified personal register such as the UK electoral roll. It is argued that the more open and trusting US business culture also makes organisations more willing to share data and trust others not to abuse granted access rights. The demand for CDI services is increased by the exceptional mobility of USA consumers and returns on investment are improved by both the very large size of client files, compared with those in other countries, and the comparatively low cost of computer processing. It is also argued that the comparative lack of loyalty of US consumers and the high level of competition results in organisations having a narrower and less deep knowledge of their customers as compared with European organisations, and hence to the greater reliance on external data.

Other than in the USA CDI systems are currently being introduced in the UK by Experian and Acxiom and in Australia by Acxiom. The takeup in the UK has largely been in the development of universal prospect databases for high-volume credit card mailers, which want to be able to consolidate mailing history into a single consistent database organised around a combination of the electoral roll and the postal address file, rather than to support real-time channels. According to Experian UK prospects are more interested in customised 'project' than standard 'product' solutions.

Unlike in the USA, where name and address changes can be sourced from a number of public and proprietary registers, it had been assumed that UK-based CDI systems will not initially be persistent — in other words the numeric key associated with a person or household would not move with them to a new address. This is because of the low level of information on movers from available reference files.

The introduction of a persistent numeric match key system, whether in Australia or the UK, could be created on the back of a client user group willing to share such information and large enough to cover the majority of house movers.

The requirement to form a club to incorporate persistency into CDI systems outside the UK, the relative paucity of base reference files in continental Europe and the less open attitude towards the exchange of personal information suggests that CDI services, perhaps like geodemographic classifications and lifestyle databases, will become a further differentiator of database marketing practice between the AngloSaxon and other Western markets. 
Perhaps the biggest difference between the USA and the rest of the Anglo-Saxon bloc is regulatory ethos. Outside the USA the most common attitude towards ID linkage is whether or not it compromises data protection legislation. In the USA, by contrast, public pressure is for online access to consumer information, a demand that data held about an individual should be fully accurate and that the consumer should be entitled to control how and when they are communicated with. Expectations of this sort cannot be satisfied without using new technologies such as ID linkage to remove inaccuracies from existing data records.

\section{References}

1. Forrester (1999) Sizing App Hosting, Forrester Research, December.

2. Hewson, W. (2002) 'Outsourced CRM: Why it will increasingly rely on application service providers', Journal of Interactive Marketing, Vol. 3, No. 3, pp. 230-242.

3. Telephone interview with Mike Wallis, Abilitech product manager, Acxiom, January 2002.

4. Telephone interview with Bruce Carroll, company leader, Corporate Marketing and Strategic Development, Acxiom, January 2002.

5. Telephone interview with Chandos Quill, Truvue product manager, Experian, January 2002.

6. Carroll, ref. 4 above

7. Jones, G. (2001) 'E-commerce and identity fraud', Journal of Interactive Marketing, Vol. 2, No. 4, pp. $357-372$. 